\title{
Budd-Chiari syndrome as the major thrombotic complication of systemic lupus erythematosus with the lupus anticoagulant
}

\author{
MORDECHAI AVERBUCH AND YORAM LEVO \\ From the Department of Medicine ' $T$ ', Ichilov Hospital, Tel Aviv Medical Center, and the Sackler School of \\ Medicine, Tel Aviv University, Tel Aviv, Israel
}

SUMmARY A patient with systemic lupus erythematosus was first diagnosed after she had developed the Budd-Chiari syndrome. We believe that the Budd-Chiari syndrome was part of her tendency for thrombosis, induced by the presence of a lupus anticoagulant. The association between enhanced thrombosis and the lupus anticoagulant is discussed, and the need for prompt recognition and administration of anticoagulant therapy is emphasised.

Key words: circulating anticoagulant, venous thrombosis.

Lupus anticoagulant (LAC) is an immunoglobulin which inhibits the conversion of prothrombin to thrombin. ${ }^{12}$ In spite of in vitro prolongation of the partial thromboplastin time (PTT), paradoxically, its presence has been associated with increased tendency to thromboembolic events and not with an increased bleeding tendency. ${ }^{3-7}$

The anticoagulant is characteristically found in patients with systemic lupus erythematosus (SLE) but has also been observed in patients with other autoimmune diseases, drug induced SLE, malignancy, and no apparent underlying disease..$^{7-11}$

Among the reported thrombotic complications of LAC only one patient with the Budd-Chiari syndrome has been described. ${ }^{12}$ This patient did not fulfil the accepted criteria of SLE. ${ }^{13}$

Herein, we report a patient with SLE who developed the Budd-Chiari syndrome after a thrombotic occlusion of the inferior vena cava associated with the presence of a circulating anticoagulant.

\section{Case report}

A 33 year old woman was admitted to our

Accepted for publication 11 November 1985.

Correspondence to Professor Yoram Levo. Department of Medicine 'T', Ichilov Hospital, 6 Weizmann Street. Tel Aviv 64239. Isracl. department of medicine in July 1984 for evaluation of arthralgias and abdominal pain. The patient was known to have had liver cirrhosis with ascites due to the Budd-Chiari syndrome for the last four years. The patient was completely healthy until the age of 24. At that time, May 1974, she first developed deep thrombophlebitis of her right calf. During the next six years she suffered from recurrent episodes of superficial thrombophlebitis of both legs, easy bruisability, menometrorrhagia, and recurrent episodes of purpura and bleeding from her gums. On repeat blood tests she was found to have thrombocytopenia of $30000-60000$ platelets $/ \mathrm{mm}^{3}$ $\left(30-60 \times 10^{9} / 1\right)$, a prolonged PTT in the presence of a normal prothrombin time, positive tests for antinuclear and anti-DNA antibodies, and an occasional hypocomplementaemia. In March 1978 the presence of a circulating anticoagulant was first diagnosed after inability to correct the patient's prolonged PTT by normal plasma. In December 1977 the patient first complained of arthralgias, and later she occasionally suffered from overt arthritis involving mainly the wrists, knees, and ankles. In January 1980 rapid enlargement of the abdomen was noted, accompanied by deterioration of liver function tests'. Subsequently, the diagnosis of the Budd-Chiari syndrome was established by a liver biopsy and venography, which showed severe 
narrowing of the inferior vena cava due to thrombotic occlusion and recanalisation just below the diaphragm, with a pressure gradient of 29 $\mathrm{mmHg}$ and non-visualisation of the hepatic veins.

Over the following three years the patient was maintained on diuretics only. On her latest admission she had evidence of ascites and a distended periumbilical venous network. The liver was not enlarged and the spleen was palpable $4 \mathrm{~cm}$ below the costal margin. The relevant laboratory data were: anaemia with a haemoglobin of $11.2 \mathrm{~g} / \mathrm{dl}$ $(112 \mathrm{~g} / \mathrm{l})$, thrombocytopenia 92000 platelets $/ \mathrm{mm}^{3}$ $\left(92 \times 10^{9} / \mathrm{l}\right)$, erythrocyte sedimentation rate $100 \mathrm{~mm} /$ $1 \mathrm{st} \mathrm{h}$, and slightly abnormal liver function tests, alkaline phosphatase $125 \mathrm{IU} / \mathrm{ml}$ (normal value $<80$ $\mathrm{IU} / \mathrm{ml}$ ), and serum aspartate transaminase $70 \mathrm{IU} / \mathrm{ml}$ (normal value $<40 \mathrm{IU} / \mathrm{ml}$ ). The serum concentrations of albumin and globulin were normal; protein electrophoresis, however, showed an increased fraction of gammaglobulin, $29.8 \%$ (normal up to $20 \%$ ). IgA and IgM blood levels were normal, with a moderately increased IgG, $2040 \mathrm{mg} / \mathrm{dl}(20.4 \mathrm{~g} / \mathrm{l})$ (normal up to $1600 \mathrm{mg} / \mathrm{dl}(16 \mathrm{~g} / \mathrm{l})$ ). The direct Coombs' test was positive, as were the tests for antinuclear and anti-DNA antibodies. The Venereal Disease Research Laboratory test (VDRL) was negative. The prothrombin time was normal in the presence of a prolonged PTT, $73 \mathrm{~min}$ (normal up to $55 \mathrm{~min}$ ). The test for LAC was repeatedly positive.

In view of the arthritis, thrombocytopenia, Coombs' positive test, anaemia and raised titres of antinuclear and anti-DNA antibodies the diagnosis of SLE in this patient with the Budd-Chiari syndrome was established. In view of the minimal evidence for SLE activity corticosteroids or cytotoxic agents were not considered to be indicated; chronic therapy with anticoagulants was recommended.

\section{Discussion}

The lupus circulating anticoagulant first described by Conley and Hartmann ${ }^{14}$ in 1952 has been recently associated with an increased tendency to develop thromboembolic phenomena. ${ }^{3-711}$ Up to 1980 only very few cases had been reported in the literature. In 1980 Mueh et al reported clinically significant thrombosis in eight out of 35 patients with the lupus anticoagulant. ${ }^{7}$ Carreras and Vermylen found eight patients with a history of thrombosis among 14 with the anticoagulant. ${ }^{15}$ Boey et al have observed thrombotic episodes in 18 of 31 patients with the anticoagulant ( 25 of these patients had SLE): 11 patients had deep vein thrombosis, 10-cerebrovascular accidents, three-pulmonary emboli, two-axillary vein thrombosis, two-central retinal vein thrombosis, and one-renal vein thrombosis. ${ }^{4}$ In another series, which included 35 patients with the anticoagulant, nine of 18 patients with SLE and $\bar{F}$ 10 of 17 non-SLE patients had single or recurrent thrombotic episodes. ${ }^{16}$ Similar data have been recently published by Jungers et al..$^{5}$ They found thrombotic episodes in 20 out of 29 SLE patients with the anticoagulant: 15 patients had a history of one or more arterial thrombotic episodes: nine had is central nervous system (CNS) involvement, two- $\overrightarrow{0}$ coronary artery thrombosis, and two visceral infarctions involving the pancreas or the spleen, seven patients had one or more episodes of deep vein thrombosis.

The Budd-Chiari syndrome is a known of complication of venous thrombosis: however, to the $i r$ best of our knowledge its association with SLE has as yet not been reported. The association of this 0 syndrome with the lupus anticoagulant has been reported in one patient only during $1984 .^{12}$ The $\vec{r}$ patient, a 37 year old man, first presented with abdominal pain and massive ascites and did not satisfy the criteria proposed for the diagnosis of $\overrightarrow{0}$ SLE. ${ }^{13}$ In contrast, our patient, in retrospect, had fulfilled the required diagnostic criteria of SLE in 1978. At that time she had already developed arthritis, thrombocytopenia, and anti-DNA antibodies, as well as a circulating anticoagulant. Her thrombotic complications first started with a deep vein thrombosis of the leg at the age of 24 in 1974, and in January 1980 she developed the Budd-Chiari syndrome. Unfortunately, the diagnosis of SLE was not established till her last admission in July 1984.

The presence of the lupus anticoagulant has also been associated with an increased incidence of thrombocytopenia, biological false positive VDRL tests, recurrent abortions, and possibly CNS and peripheral nerve involvement. ${ }^{4-6} 1116$ Recent studies have suggested that the anticoagulant reacts with a phospholipid related antigen shared by clotting factors, platelets, cardiolipin, and other cell 0 membranes. ${ }^{46711}$ The reaction between the antibody and the antigen could result, on the one $\sigma$ hand, in inhibition of clotting factors and $N$ thrombocytopenia, and on the other hand, in N inhibition of prostacyclin production, release of procoagulant activity, and enhanced thrombosis. 6111516

In view of the severe thrombotic complications in patients with the lupus anticoagulant it has been suggested that these patients should be treated with 0 anticoagulants. ${ }^{11}$ In fact, Pomeroy et al treated their $\mathbb{\mathbb { D }}$ patient with the Budd-Chiari syndrome with $\frac{O}{\mathbb{D}}$ coumadin and reported resolution of ascites attributed to recanalisation, with no recurrent 
ascites over a one year follow up. ${ }^{12}$ Other therapeutic approaches include administration of corticosteroids and cytotoxic agents and plasmapheresis in order to decrease the serum level of the anticoagulant. ${ }^{45} 11$ Jungers $e t$ al have given corticosteroid therapy to 23 patients. ${ }^{5}$ In 13 patients a decrease in anticoagulant level was noted, in parallel with the reduction of lupus activity.

To conclude, the Budd-Chiari syndrome should be included among the thrombotic complications of SLE patients with a circulating anticoagulant. Prompt recognition and administration of anticoagulant therapy should improve the prognosis of such patients. In addition, one should consider the possibility that lupus type circulating anticoagulants may have a pathogenic mechanism in the 'idiopathic' Budd-Chiari syndrome, and therefore all patients with the syndrome should be screened for the presence of such an anticoagulant even in the absence of any other evidence of SLE.

\section{References}

1 Feinstcin D I, Rapaport S I. Acquired inhibitors of blood coagulation. Prog Hemost Thromb 1972; 1: 75-95.

2 Exner T, Rickard A. Kroenberg H. Studies on phospholipid in the action of lupus coagulation inhibitors. Pathology 1975; 7: 319-28.

3 Bowie E J W, Thompson J H, Pascuzzi C A, et al. Thrombosis in systemic lupus erythematosus despite circulating anticoagulants. J Lab Clin Med 1963; 62: 416-30.
4 Boey M L, Colaco C B, Gharavi A E, Elkon K B, Loizou S, Hughes G R V. Thrombosis in systemic lupus erythematosus: striking association with the presence of circulating lupus anticoagulant. $\mathrm{Br}$ Med J 1983; 287: 1021-3.

5 Jungers P, Liote F, Dautzenberg M D, et al. Lupus anticoagulant and thrombosis in systemic lupus erythematosus. Lancet 1984: i: 574-5.

6 Carreras L O, Machin S J. Deman R, et al. Arterial thrombosis. intra-uterine death and lupus anticoagulant: detection of immunoglobulin interfering with prostacyclin formation. Lancet 1981; i: 244-6.

7 Mueh J R, Herbst K D, Rapaport S I. Thrombosis in patients with lupus anticoagulant. Ann Intern Med 1980; 92: 156-9.

8 Schleider M A, Nachman R L, Jaffee E A, et al. A clinical study of the lupus anticoagulant. Blood 1976; 48: 499-509.

9 Lechner K. Acquired inhibitors in non-hemophiliac patients. Haemostasis 1974: 3: 65-93.

10 Boxer M, Ellman L. Carvalho A. The lupus anticoagulant. Arthritis Rheum 1976; 19: 1244-8.

11 Byron M A. The clotting defect in SLE. Clin Rheum Dis 1982; 8: $137-51$.

12 Pomeroy C, Knodell R G, Swain W R. Arneson P, Mahowald $M$ L. Budd-Chiari syndrome in a patient with the lupus anticoagulant. Gastroenterology 1984; 86: 158-61.

13 Tan E M, Cohen A S. Fries J F, et al. The 1982 revised criteria for the classification of systemic lupus erythematosus. Arthritis Rheum 1982; 25: 1271-7.

14 Conley C L. Hartmann R C. A hemorrhagic disorder caused by circulating anticoagulant in patients with disseminated lupus erythematosus. J Clin Invest 1952: 31: 621-2.

15 Carreras L O, Vermylen J G. 'Lupus' anticoagulant and thrombosis: possible role of inhibition of prostacyclin formation. Thromb Haemost 1982; 48: 38-40.

16 Elias M, Eldor A. Thromboembolism in patients with the 'lupus'-type circulating anticoagulant. Arch Intern Med 1984; 144: $510-5$ 